\title{
BMJ Global Health mHealth text and voice communication for monitoring people with chronic diseases in low-resource settings: a realist review
}

\author{
Jocelyn Anstey Watkins, ${ }^{1}$ Jane Goudge, ${ }^{2}$ Francesc Xavier Gómez-Olivé, ${ }^{3}$ \\ Caroline Huxley, ${ }^{1}$ Katherine Dodd, ${ }^{1}$ Frances Griffiths ${ }^{1,2}$
}

To cite: Anstey Watkins J, Goudge J, Gómez-Olivé FX, et al. mHealth text and voice communication for monitoring people with chronic diseases in low-resource settings: a realist review. BMJ Glob Health 2018;3:e00543. doi:10.1136/ bmjgh-2017-000543

Handling editor Valery Ridde

Received 30 August 2017 Revised 7 February 2018 Accepted 9 February 2018

Check for updates

${ }^{1}$ Division of Health Sciences, Warwick Medical School, University of Warwick, Coventry, UK

${ }^{2}$ Centre for Health Policy, Faculty of Health Sciences, School of Public Health, University of the Witwatersrand, Johannesburg, South Africa

${ }^{3} \mathrm{MRC} /$ Wits Rural Public Health and Health Transitions Research Unit (Agincourt), School of Public Health, Faculty of Health Sciences, University of the Witwatersrand, Johannesburg, South Africa

Correspondence to Dr Jocelyn Anstey Watkins; jotawatkins@gmail.com

\section{ABSTRACT}

Background Routine monitoring by patients and healthcare providers to manage chronic disease is vital, though this can be challenging in low-resourced health systems. Mobile health (mHealth) has been proposed as one way to improve management of chronic diseases. Past mHealth reviews have proposed the need for a greater understanding around how the theoretical constructs in mHealth interventions actually work. In response, we synthesised evidence from primary studies on monitoring of chronic diseases using two-way digital text or voice communication between a patient and health worker. We did this in order to understand the important considerations for the design of mHealth interventions.

Method Articles retrieved were systematically screened and analysed to elicit explanations of mHealth monitoring interventions. These explanations were consolidated into programme theory and compared with existing theory and frameworks. We identified variation in outcomes to understand how context moderates the outcome.

Results Four articles were identified - monitoring of hypertension and HIV/AIDS from: Kenya, Pakistan, Honduras and Mexico and South Africa. Six components were found in all four interventions: reminders, patient observation of health state, motivational education/advice, provision of support communication, targeted actions and praise and encouragement. Intervention components were mapped to existing frameworks and theory. Variation in outcome identified in subgroup analysis suggests greater impact is achieved with certain patient groups, such as those with low literacy, those with stressful life events or those early in the disease trajectory. There was no other evidence in the included studies of the effect of context on the intervention and outcome.

Conclusion mHealth interventions for monitoring chronic disease in low-resource settings, based on existing frameworks and theory, can be effective. A match between what the intervention provides and the needs or social factors relevant to specific patient group increases the effect. It was not possible to understand the impact of context on intervention and outcome beyond these patientlevel measures as no evidence was provided in the study reports.

\section{Key questions}

What is already known about this topic?

- The burden of chronic diseases is an escalating problem globally. Evidence reviews suggest mHealth interventions delivered in low-income and middle-income countries can be effective in improving health outcome for people living with chronic disease.

What are the new findings?

- Effective interventions using two-way digital communication between healthcare providers and patients living with long-term conditions are based on established frameworks and behaviour change theory.

- Outcome is improved for patients with low literacy, stress-inducing life events and being recently diagnosed.

- Contextual factors beyond those measured at patient level were not reported in the studies.

Recommendations for policy

- mHealth interventions for improving the monitoring of chronic disease, when based on existing frameworks and theory, have potentia for improving patient care and health outcome particularly when tailored to the needs of specific patient groups

\section{INTRODUCTION}

The burden of chronic diseases is an escalating problem in low-income and middle-income countries (LMICs). ${ }^{1}$ The Sustainable Development Goals state that by 2030 , improving the prevention and management of chronic communicable and non-communicable diseases is a priority for primary care in public health systems. ${ }^{23}$ Chronic diseases are long-term, disruptive and often intrusive to individuals' everyday lives. ${ }^{4}$ The management of chronic diseases is a dynamic process that varies over time, depending on the disease 


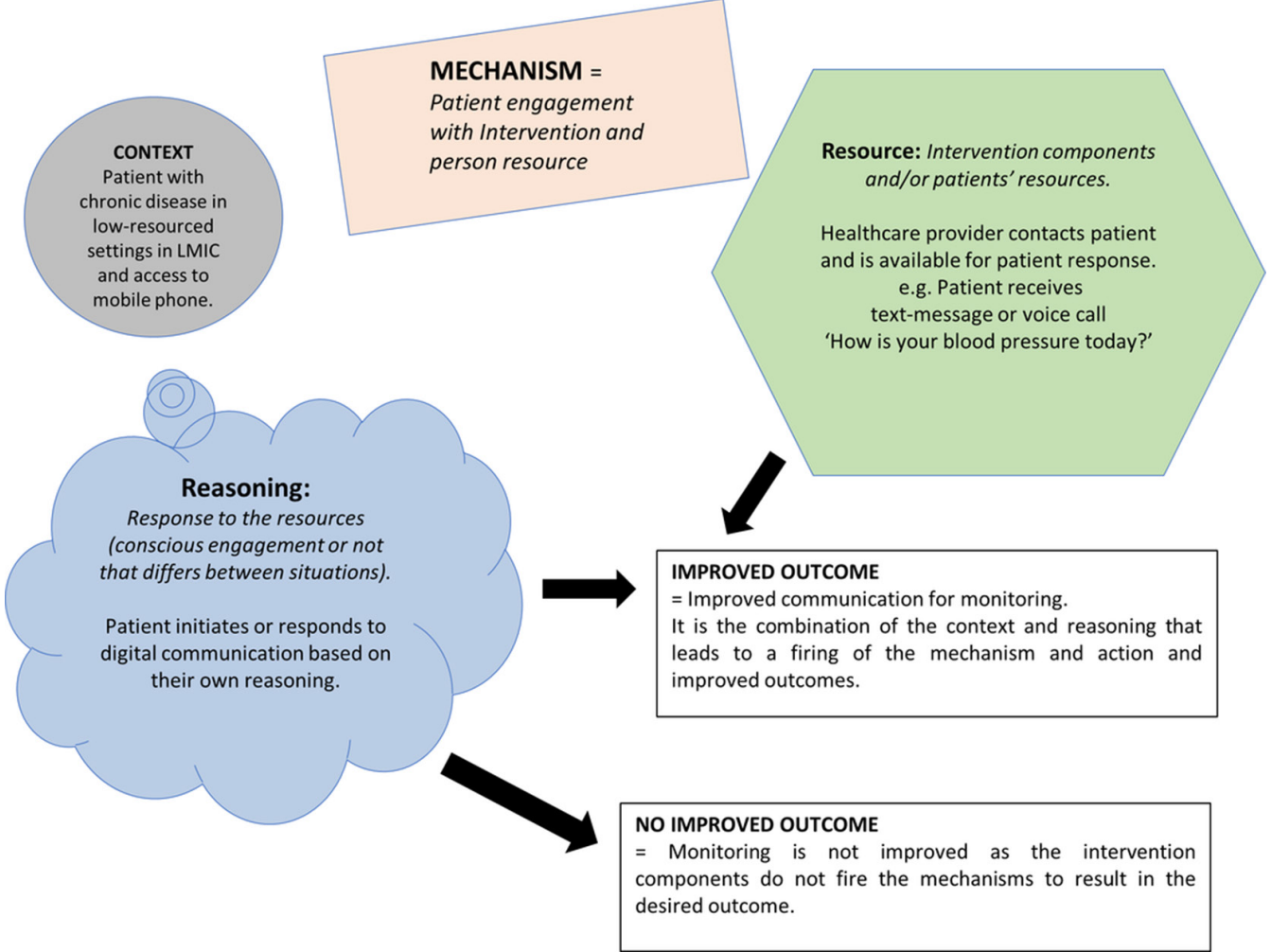

Figure $1 \mathrm{mHealth}$ example of a CMOc summarised from the reviews. CMOc, Context-Mechanism-Outcome configuration; LMIC, low-income and middle-income countries.

aetiology and physiology. ${ }^{5}$ Chronic disease involves regular self-care ${ }^{6} 7$ and routine monitoring by patients and health workers to check disease progress or regress. ${ }^{8}$ Long-term monitoring encompasses adhering to treatment and capturing vital signs or clinical indicators. The purpose is to improve outcomes and quality of life ${ }^{9}$ by reducing acute exacerbations and premature death ${ }^{10}$ and to maximise health.

The monitoring of chronic diseases can be challenging, particularly in low-resourced health systems limited by long distances to health facilities and low staff capacity. ${ }^{11}$ In this environment, mobile health (mHealth) has been proposed as an approach to improve management of chronic diseases ${ }^{12-14}$ including assistance with monitoring. mHealth technologies used in LMIC include portable wireless devices, including mobile phones and tablets. ${ }^{15}$ mHealth can involve one-way or two-way communication between the health worker and patient, using any digital channel that allows the users to be mobile. Vasudevan $e t a l^{16}$ suggest that strides are being made in strengthening the global mHealth evidence base along with the key 'best practices' in scaling mHealth for achieving universal health coverage.

A systematic search for mHealth reviews in seven databases found several $\mathrm{mHealth}$ reviews that included aspects of monitoring such as adherence studies, ${ }^{17}$ behaviour change $^{18}$ and attendance reminders. ${ }^{1920}$ Review authors described how the effectiveness of mHealth evidence is mixed. They suggest there is a lack of understanding of why mHealth interventions for chronic disease management should work (or not) in LMICs. ${ }^{17} 18$ 20-27 One of these reviews proposed that future studies should explicitly describe the theoretical constructs that mHealth interventions are targeting. ${ }^{28}$ They argue this will make it clearer how and why the intervention is intended to work and in what circumstances. ${ }^{28} \mathrm{~A}$ stronger theoretical understanding is likely to strengthen the mHealth evidence base. ${ }^{27}$ However, this search revealed that there is no systematic review set in LMICs specifically focusing on one-way and two-way mHealth communication for monitoring of chronic disease.

This paper responds to the challenge identified in the systematic reviews, by examining the theoretical foundations and mechanisms by which mHealth interventions work (or not), to support chronic disease monitoring in LMICs.

We focus on understanding how monitoring of chronic diseases may be improved using two-way digital communication between a patient and health worker (community health worker/nurse/doctor) in a low-resourced setting to potentially guide future intervention design.

\section{METHOD}

\section{The realist method and initial search}

A 'realist review', also known as a 'realist synthesis', makes sense of heterogeneous evidence about complex interventions applied in diverse contexts. ${ }^{29}{ }^{30}$ It draws on substantive theory and empirical research from across multiple disciplines ${ }^{31}$ to understand how interventions 
Table 1 Databases, search terms and inclusion and exclusion criteria for identifying empirical studies

$\begin{array}{ll}\begin{array}{l}\text { Databases } \\ \text { searched }\end{array} & \text { Cochrane Register of Controlled Trials, Medline (Ovid), PubMed, Web of Science, Psych Info, } \\ & \text { Google Scholar, Knowledge for Health }\end{array}$

Search terms Patient* AND low- and middle- income countr* OR LMIC OR developing* AND chronic disease* AND mobile phone* OR text messag* OR SMS OR voice AND monitor ${ }^{\star}$ OR self-monitor ${ }^{\star}$ OR self-manage*

Inclusion criteria Population: Patients with any chronic disease and all cadres of health workers in a public health system. Context: Low-resourced settings of any LMICs.

Intervention design: The study interventions include two-way exchange of digital information necessary for monitoring initiated by the patient or health worker. The mHealth resource is either voice call or text message based mobile phone communication to improve monitoring of chronic disease.

Monitoring: monitoring of side effects of medication, monitoring of physiological measures such as blood pressure or monitoring of how well the patient feels and offering relevant information and support and reminding them about future appointments or medication compliance.

Study design: Empirical research evaluating the effectiveness of the intervention (any design). The study includes a description of the intervention.

Outcomes: Impact/health and process outcomes (primary and secondary).

$\begin{aligned} \text { Exclusion criteria } & \text { Studies set in high-income countries. } \\ & \text { Studies using only landline telephones or computer-based communication. } \\ & \text { Protocols or reports of intervention development with no published evidence outcome. } \\ & \text { Seasibility and pilot studies with no outcome for the intervention. } \\ & \text { Reviews and reviews of reviews. }\end{aligned}$

LMICs, low-income and middle-income countries.

work and for whom. By using the methods of a realist review, ${ }^{32-34}$ we questioned what works, compared with what, how well, with what exposure, with what behaviours, for how long, for whom, in what settings and why ${ }^{35}$ ? We went through the following realist stages: (1) identifying programme theory, how the study authors intended their interventions to work; (2) testing this programme theory against empirical evidence and established highlevel theory and (3) providing guidance for future intervention development.

We did a preliminary systematic search for systematic reviews of mHealth. From the 10 relevant reviews identified, ${ }^{17} 18$ 20-27 we extracted examples of how the review authors thought the interventions worked from their included papers. We mapped these on to the layout of the realist Context-Mechanism-Outcome configuration (CMOc) and adapted the diagram designed by Dalkin ${ }^{36}$ (figure 1) using the example of mHealth monitoring. Figure 1 illustrates how mechanisms are defined as the reactions or responses to the resources available within an interaction process that leads to outcomes. ${ }^{37}$ Mechanisms are the responses to the intervention or patients' resources and are contingent and conditional, only firing in particular contexts. ${ }^{38}$ The outcomes are the desired response to the resources resulting from the participants' reasoning ${ }^{39}$ within a particular context.

\section{Main search to identify empirical papers}

To identify relevant empirical studies, we conducted the main search to systematically find papers on the monitoring of chronic disease using two-way text message or voice call interventions in LMICs, written in English, published from January 2000 to March 2017. We limited the search to the year 2000 or later as mobile phones were not widely available before then. ${ }^{28}$ Seven appropriate databases and 12 search terms were used. Titles and abstracts were screened against the inclusion and exclusion criteria, as detailed in table 1 . Each reference list of the final included papers in this review was checked to identify further relevant primary research studies.

\section{Data extraction from empirical papers}

From the included studies, we extracted data (table 2) on: Research design, participant sample, setting, outcome measures; intervention description, components and study duration; Intervention effect; Authors' programme theory where stated and our interpretation of whether the study worked as intended.

Where authors did not describe how they thought their intervention would work, we worked this out from careful reading of the description of the intervention. We also referred to related study protocols, if available, or other accompanying papers such as process evaluations of the trial. JAW, FG and KD extracted data independently and then compared data to resolve any inconsistencies.

\section{Identifying established theory to test the programme theory}

We used the CMOc in the diagram in figure 1 and applied this to each empirical study on chronic disease monitoring found in the search. ${ }^{33}$ We developed a summary of how the interventions were intended to work according to the study authors. We then searched for relevant established high-level theory related to study intervention components and through further literature searching. As most of the mechanisms were behaviour-based, we looked mostly to psychology for the theory. We aimed to match established theory to each intervention component type (table 3) to help us reach our final CMOc. The theories 


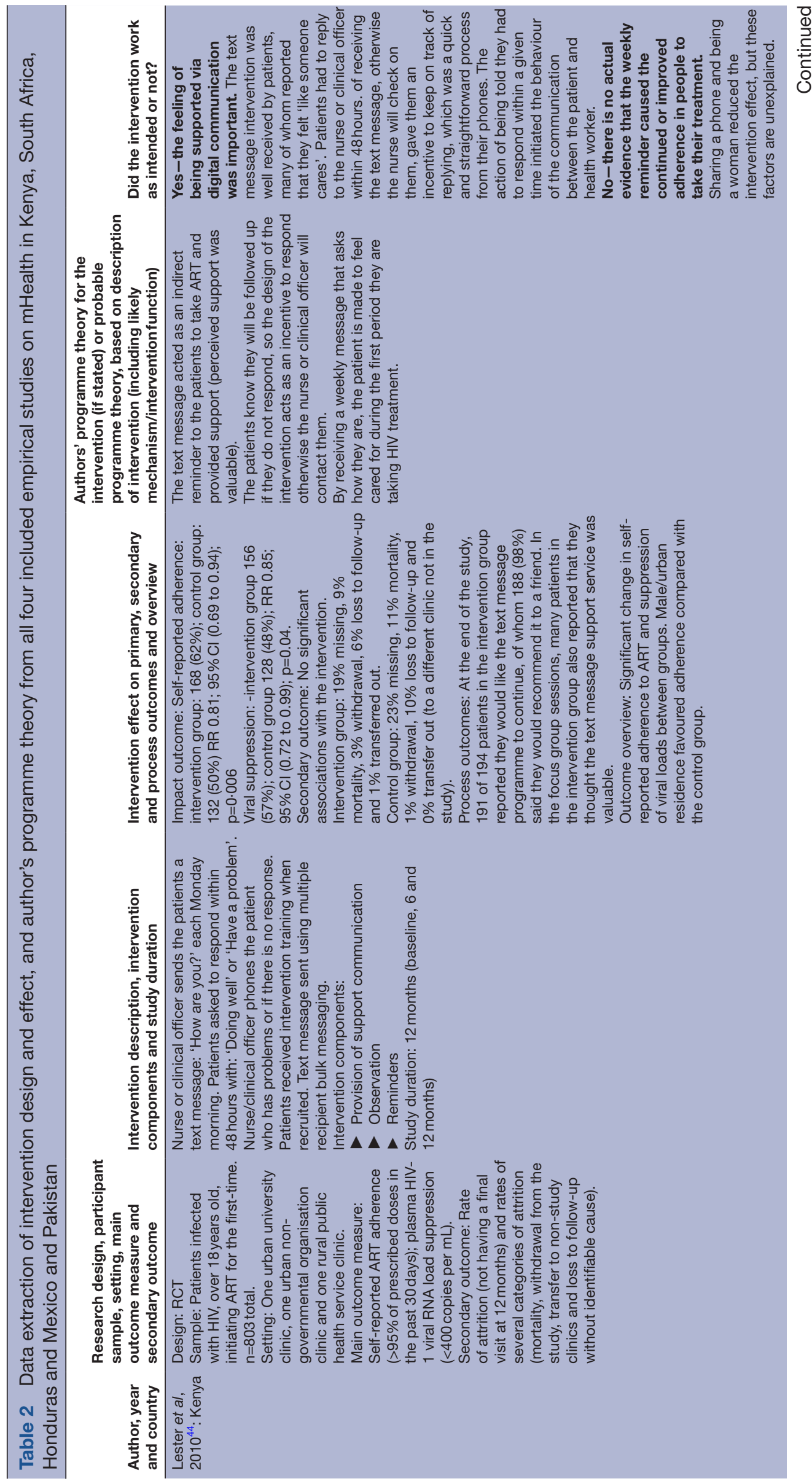




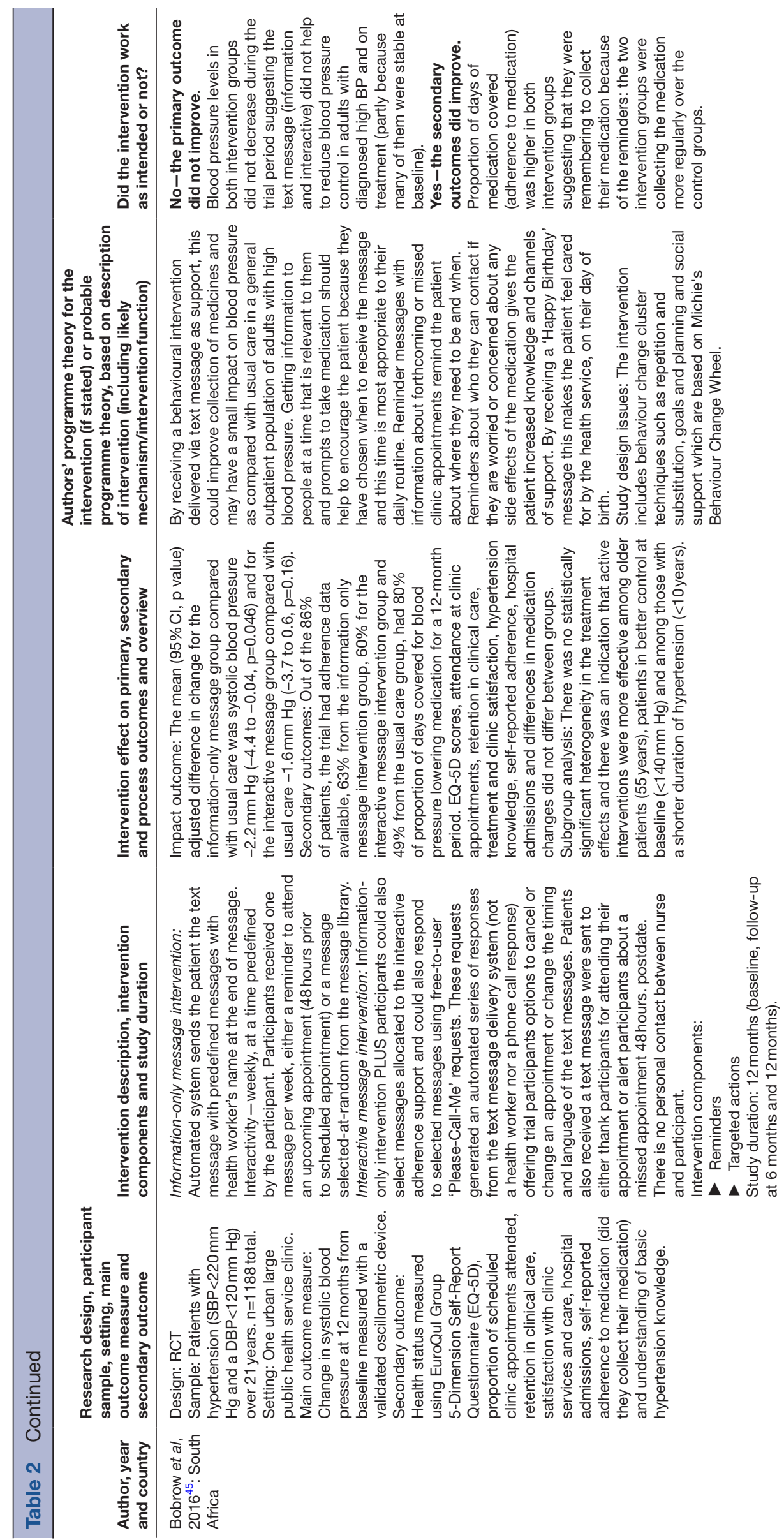

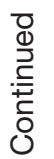




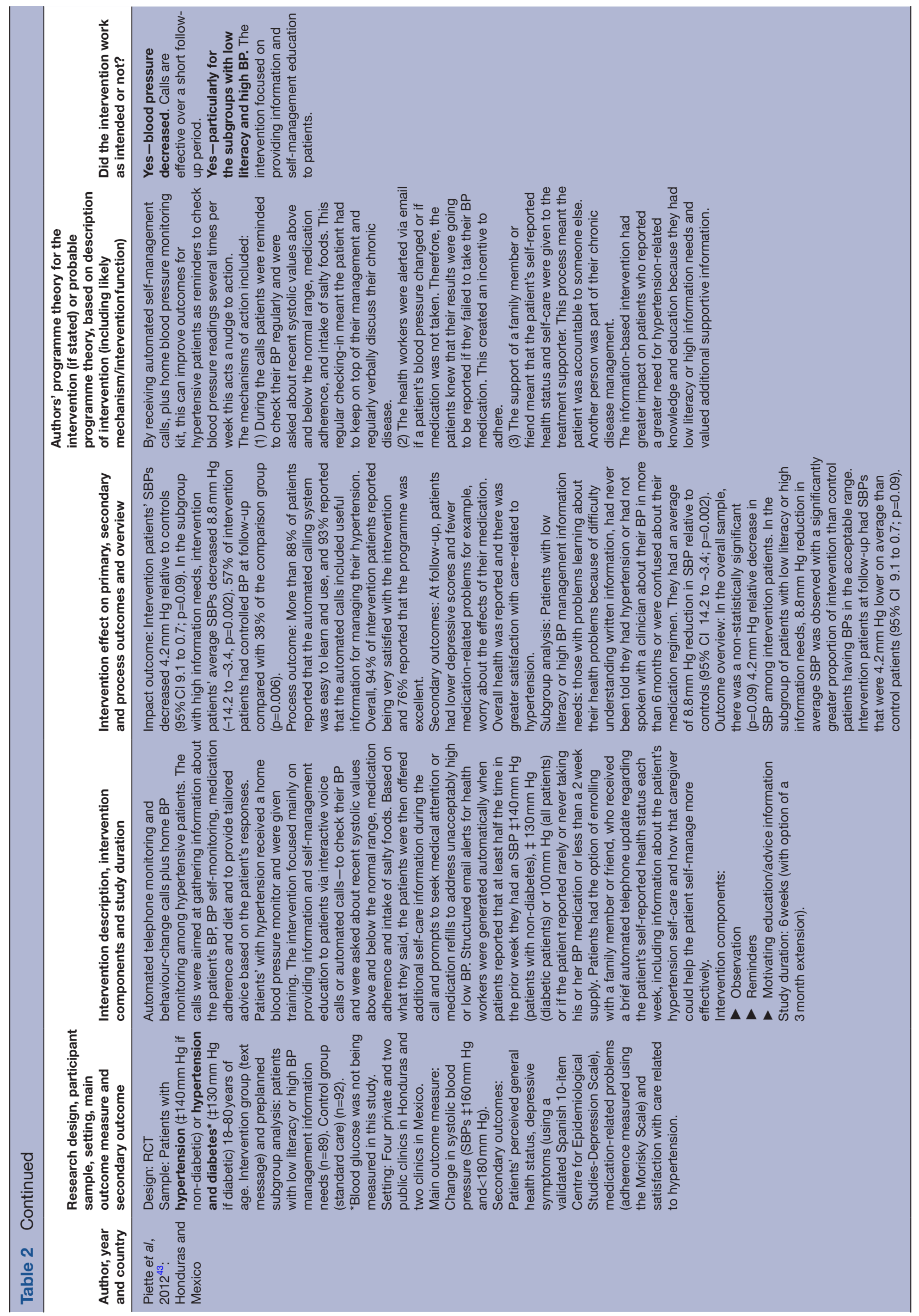




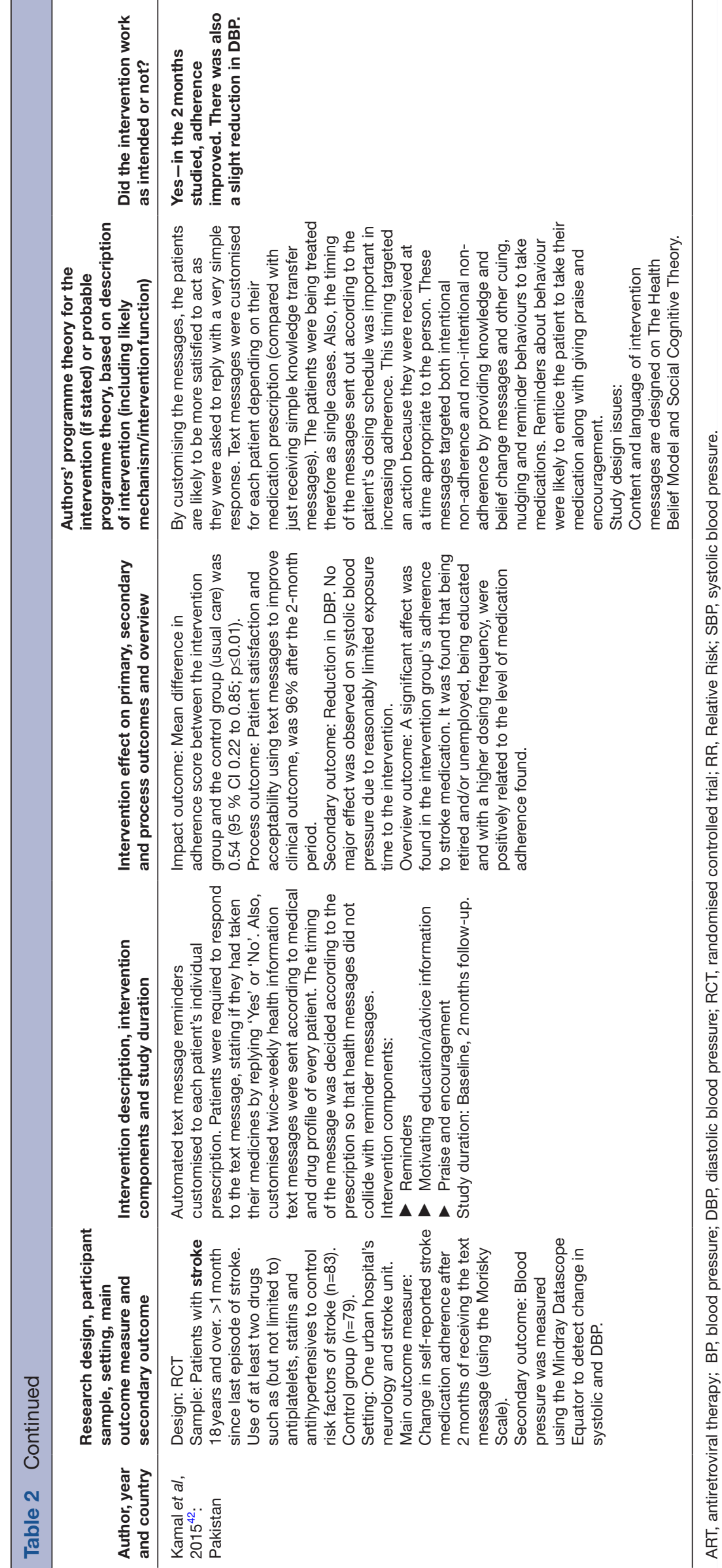




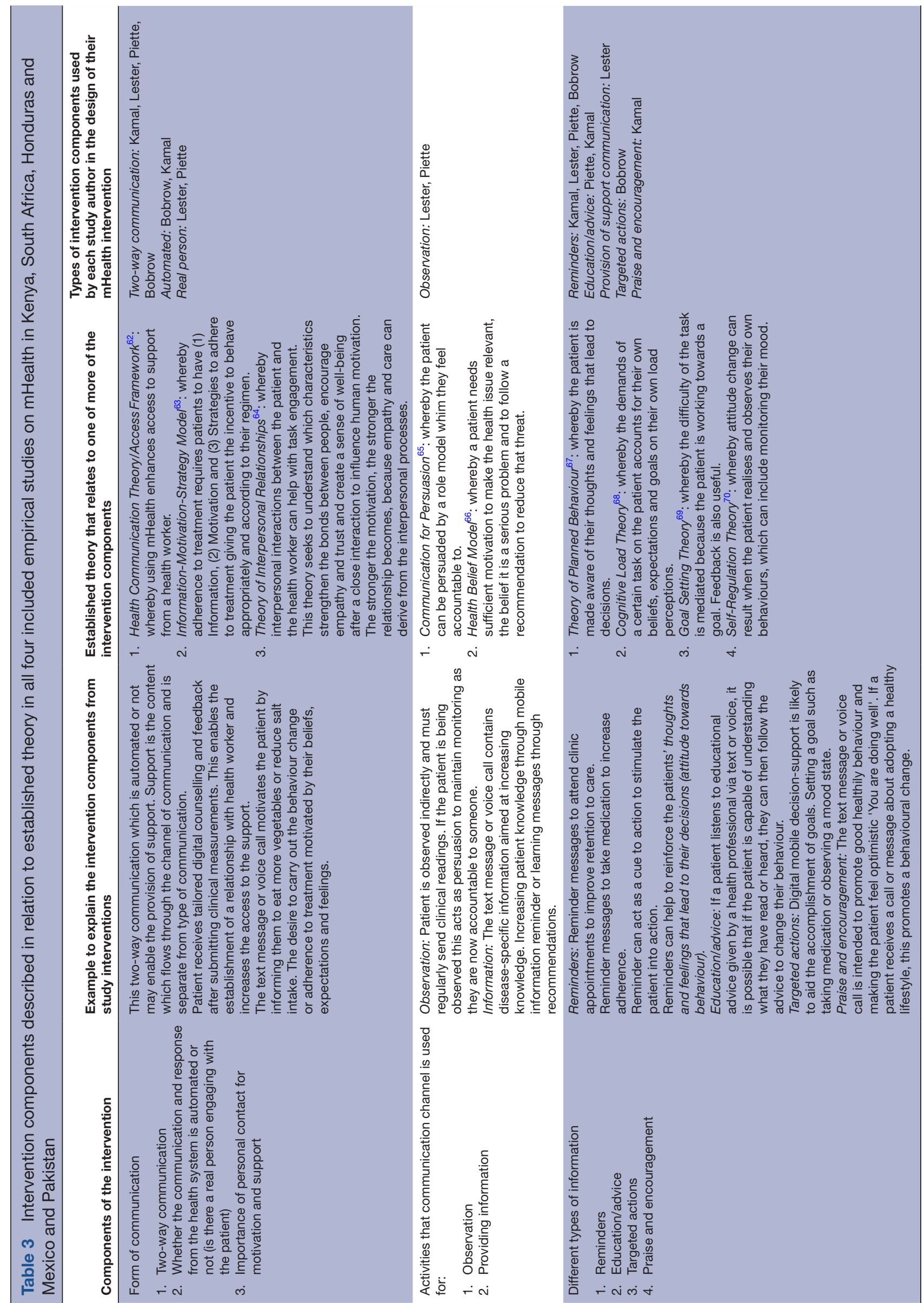


helped us to understand how and why each study's intervention design and possible components of the intervention may have contributed to the study being effective or not. These theories were then used to help us search for higher-level frameworks (table 4) based on similar theories to those described in table 3 .

We found two frameworks to use in our analysis that brought together mechanisms and theories:

- COM-B framework, ${ }^{40}$ designed for Behaviour change, describes the three domains of: Capability: physical skills, knowledge, behavioural regulation and memory, attention and decision process; Opportunity: environmental context, resources and social influences; Motivation: beliefs about consequences, optimism and beliefs about capabilities and reinforcement and emotion.

- RFV framework, ${ }^{41}$ designed for the successful implementation of telecommunications technology health interventions, describes the three domains of: Relationships: with health workers and peer as a means of providing support for behavioural change, feedback and reinforcement; Fit: integration of mHealth into routine and its ease of use into the existing environment and Visibility: to engage in information to mediate and motivate self-management tasks and enable enhanced awareness.

COM-B domains provide a framework for understanding patient behaviours. RFV domains provide a framework to understand how mHealth interventions have been designed to be implemented. The domains of these frameworks include theory relevant to the mechanisms of action of the interventions in our review. We used these theoretical frameworks to understand what mechanisms, in what context will result in a behaviour change.

The Realist and Meta-Narrative Evidence Syntheses (RAMESES I) reporting standards for realist reviews were followed. $^{33}$

\section{RESULTS \\ Search results}

The process of screening and paper selection resulted in 57 references (PRISMA diagram in figure 2). Of those, 29 papers were assessed for full text eligibility and a total of four studies were eligible for inclusion (table 2).

\section{The studies and their design}

The four papers included studies conducted in Pakistan (Kamal), ${ }^{42}$ Honduras and Mexico (Piette), ${ }^{43}$ Kenya (Lester) ${ }^{44}$ and South Africa (Bobrow) ${ }^{45}$ The studies focused on specific diseases: stroke (Kamal), hypertension (Bobrow and Piette) and HIV (Lester). Patients were all over 18 years of age. All four study designs were randomised controlled trials with control groups receiving usual standard care, all set in urban healthcare settings. Two studies primarily measured clinical outcomes (Bobrow and Piette) and two measured self-reported adherence (Lester and Kamal). The interventions were designed to facilitate communication via mobile phones between the patient and health worker. All interventions involved medication adherence. Two studies had specific subgroup analyses; Piette's subgroup was low-literate patients with information needs for how to manage high blood pressure; Bobrow's subgroup was patients over 55 years of age who had good control of blood pressure at the baseline measure and patients on treatment for hypertension for less than 10 years.

The intervention design in three studies was text messages sent weekly to patients (Lester, Bobrow, Kamal). The other study's intervention was weekly automated phone calls (Piette) (table 2). In two studies, data submitted by patients were interpreted using an automated system (Kamal and Bobrow), whereas in the other two studies, there was engagement with a real health worker (Piette and Lester). In Bobrow's study, there was an option for the patient to use their phones to initiate a free to user 'Please Call Me' request. A health worker did not actually phone the patient back. There was no verbal communication in this instance; instead, this request generated an automated series of responses from the text message delivery system. This design offered trial participants several options including cancelling or moving an appointment, and changing the timing and language of the incoming text messages, whereas in Bobrow's and Kamal's studies, the participants predefined when they wanted to receive the message to their phone. This gave them patient choice, as the other studies contacted the patient at a time decided on by the intervention team. In the case of Kamal's study, when the intervention was described as automated, we had to assume that a health worker was reading the patient's responses and taking action, especially when the patient had a problem. This was not made explicit in the paper and so it was unclear what the health worker's role was.

Two studies (Lester and Bobrow), used standardised text messages whereas the other two (Kamal and Piette), used customised messages for each patient (table 2). In Kamal's study, each patient received tailored text messages with information content about the patients' individual prescriptions. Likewise, in Piette's study, the automated calls could be tailored as patients were directly asked to provide blood pressure measurements by using the home monitoring kit, in addition to the patient's mobile phone. In Lester's study, they used very indirect ways of assisting patients to monitor as the content of the text messages were aimed at enquiring how they were feeling, without any reference to HIV, whereas in Bobrow's trial, the information messages did include the words related to the patient's chronic disease: 'high blood pills'.

\section{Intervention components and their intended purpose}

Intervention components were similar across the studies, but the combinations of varying components used were different. Intervention components included reminders, patient observation of health state, motivational 


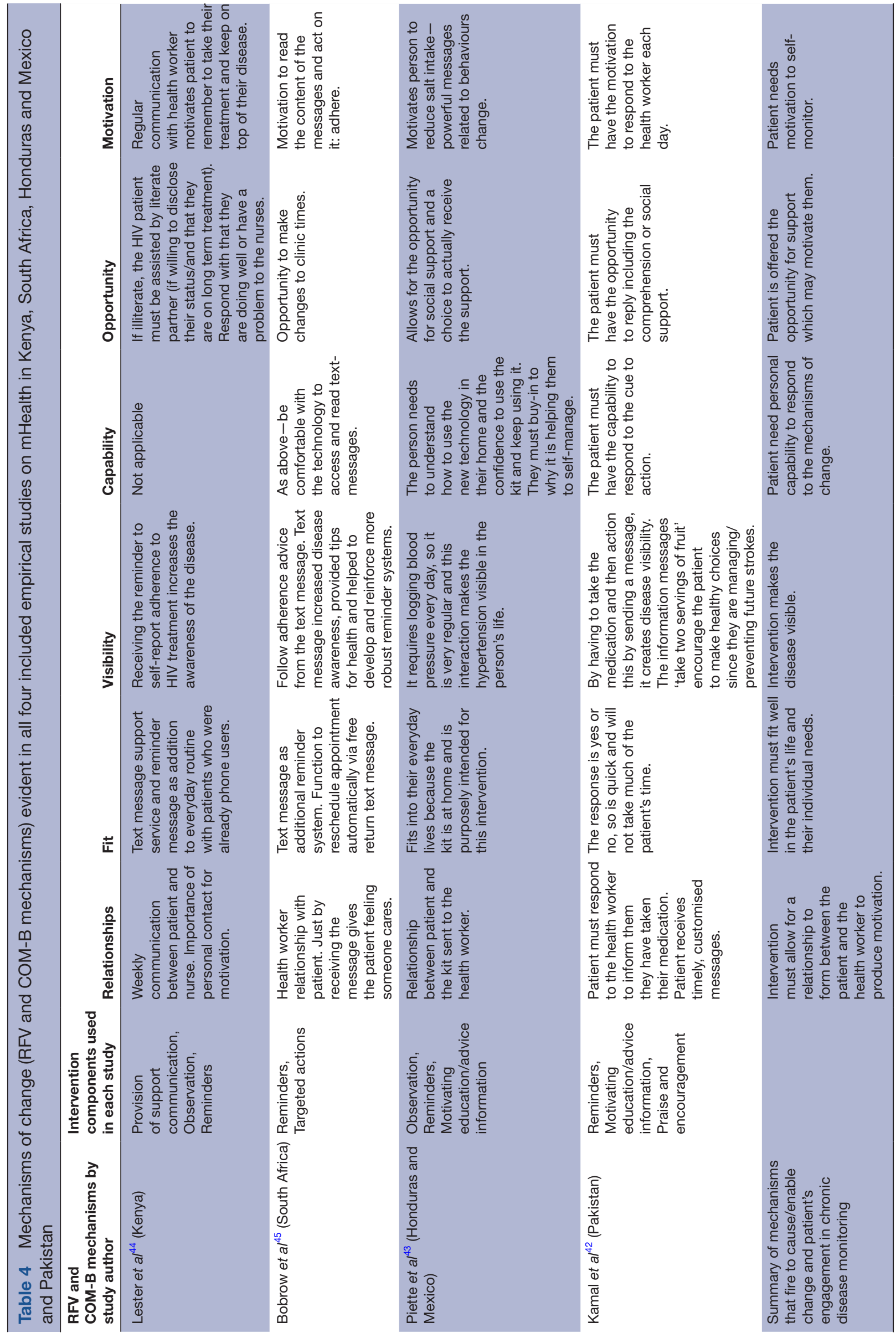




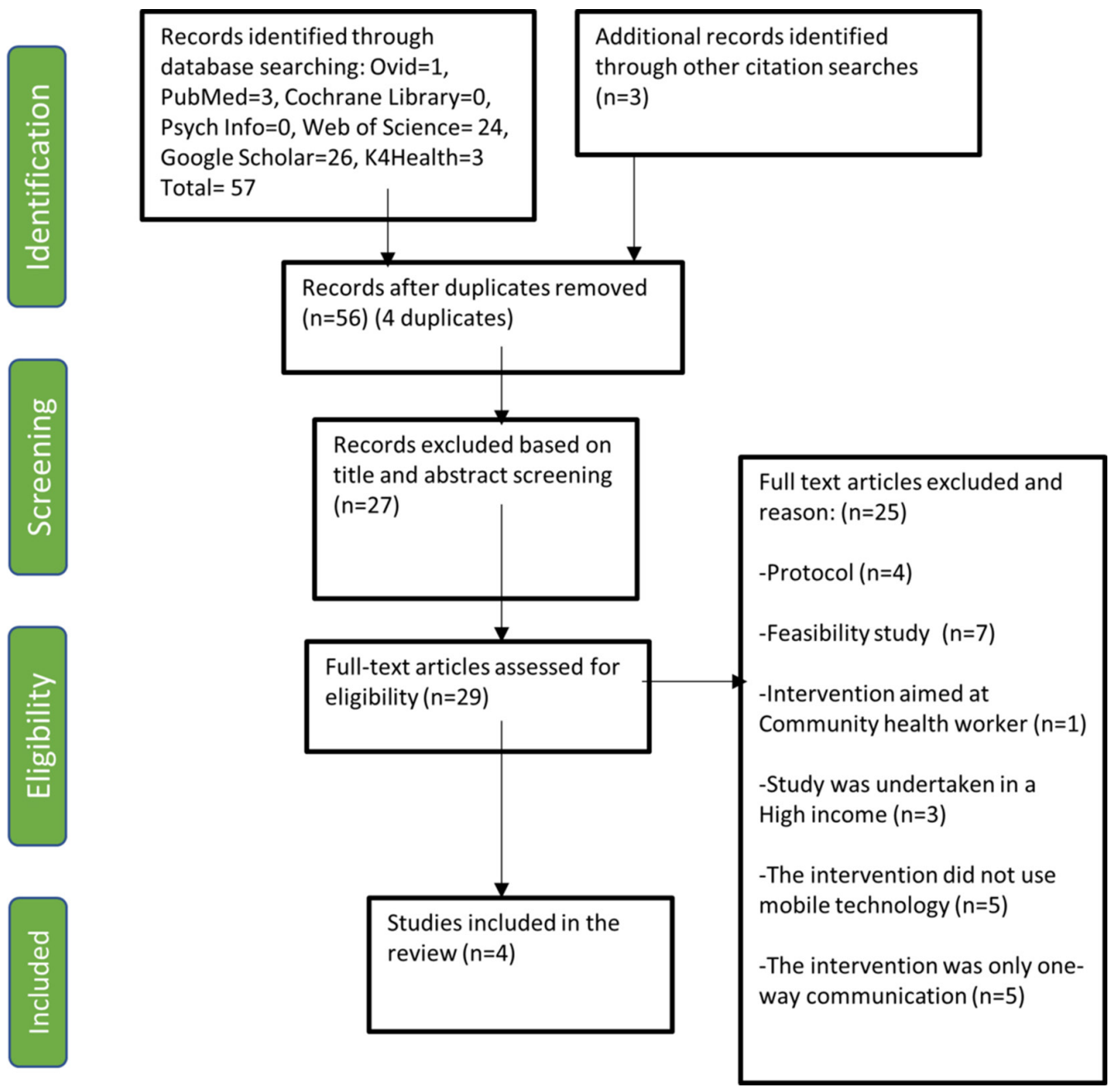

Figure 2 PRISMA.

education/advice, provision of support communication, targeted actions and praise and encouragement. Given the extracted results in table 2, in table 3, we then describe how each intervention's components relate to theory. We found this midway process useful in helping to define the programme theory.

All except Bobrow's study required a response from the patient. Instead, the two information groups received information-only messages and the second intervention group also had to the option to interact with the automated message, if desired.

\section{Programme theory: how the intervention was intended to work}

Only Bobrow's study provided a clear statement of the programme theory and how the authors intended their intervention to work (described in full, in another publication $^{46}$ ). It was supported by a process evaluation of the study in a paper by Leon. ${ }^{47}$ The other studies mentioned the theories on which they based their intervention design but did not explicitly explain why they felt their intervention worked. For Kamal and Lester's studies, we used related published study protocols or supplementary material to work out their programme theory. The programme theories are reported in table 2.

Programme theory summary from across the four included studies:

- All four studies used weekly messages/calls. This was intended to make the patient feel cared for. These included non-health related information such as 'Happy Birthday' messages (Bobrow).

- In three studies (Lester, Piette and Kamal), a non-response from the patient or the reporting of poor blood pressure readings triggered a health worker to follow-up the patient. This process of follow-up acted as incentive to the patient to always respond in the specified period (Piette).

- In two studies (Bobrow and Kamal), patients were asked, when they wanted to receive the message at a time of day that was relevant to them. This prompted the patient to take action as they had chosen to receive the communication at that specified time.

- In Piette's study, patients had to take their blood pressure measurements after a cue to action. The other three studies did not require the patient to take clinical measurements as home-monitoring kit was not 
provided. Also, in Piette's study, the patient's report was also sent to a predefined treatment supporter. This was intended to make the patient feel they were accountable to somebody who cares for them. This was the only study to use an external person beyond the patient-health worker.

- Two studies (Piette and Kamal) customised the messages to ensure the patient felt more satisfied and to prompt them into action.

- In two studies, the text messages required simple quick responses such as 'Yes or No' (Kamal) or 'I am fine' (Lester). These responses were appropriate as the patient did not have to spend much time replying.

\section{Mechanisms of action}

All four studies used the domains of RFV and COM-B as mechanisms of change to some degree (table 4).

\section{RFV}

Relationship

There needs to be personal contact within the relationship between the patient and health worker to motivate the patient or the text messages need to be tailored to make the patient feel like someone cares. Fit: The level of how well the mHealth intervention fits into the patient's daily life is crucial to how they will respond. Visibility: It appears that visibility acts as a mechanism in the studies because the mHealth component's purpose is to improve monitoring and to therefore make the disease more visible in the patients' lives, at least once a week.

\section{COM-B}

\section{Capability, Opportunity, Motivation=Behaviour}

The interpersonal relationship between the patient and health worker created when the mHealth intervention is not automated improves the patient's motivation to feel capable of responding or adhering to their treatment regimen. When the patient feels cared for by the real person who is involved, this leads to change in the patients' behaviour. When the patient is offered the opportunity to be given information that provides education and advice, this may allow them to engage with the information. This in turn may improve the way they manage their disease, as they feel more capable of doing so. Even automated communication can motivate patients to engage.

In all four studies, the patients engage with the intervention components (mHealth resources) by using their own reasoning. Their reasoning is made up of their own motivation and empowerment to respond to the resources. It is this engagement that leads to change and thus improvements in the study's primary and/ or secondary outcomes (table 2, final column). Table 4 summarises the combinations of mechanisms for each intervention.

In Lester's study, the mHealth components of provision of supportive communication, self-observation and reminders prompted engagement with a health worker (relationship). This personal contact with the nurse resulted in the patient being motivated to collect their medication from the clinic; otherwise a nurse will check-up on them. This personal contact with the nurse and feeling more supported resulted in the patient being motivated to take their treatment (table 4) and keep on top of their disease management. However, this did not lead to improved adherence in treatment.

In Bobrow's study, the mHealth components were reminders, opportunity to make changes to appointment times so the patient was able to fit the intervention into their daily life. The text messages increase disease awareness and thus its visibility. The mHealth intervention allowed the patient to feel cared for by the nurse and to take more control of their management by having the option to change appointments from their phones. This did not lead to a decrease in blood pressure; however, adherence to medication improved.

In Piette's study, reminders and motivating education/advice information prompted engagement with a health worker and treatment supporter (relationship). The patient felt they had empathetic support, especially when the patient had problems. Disease management was made more visible as the patient had to log onto the home-monitoring kit every day. Information messages prompted change in lifestyle. This regular interaction resulted in the opportunity to engage with social support when they needed it. By reading powerful practical messages, this motivated them to reduce salt intake for example. This led to a decrease in blood pressure, particularly in the subgroup.

In Kamal's study the reminders and motivating education/advice information were tailored to the individual patients making the patient feel they had a relationship with their health worker, even though the intervention did not have any direct patient-health worker communication. By having to respond to the health worker, this resulted in the quick 'Yes' or 'No' response message becoming routinised and did not take much of the patient's time. This led to improved adherence to medication and a slight reduction in diastolic blood pressure. Table 4 summarises which combinations of mechanisms help to determine what is the outcome of the mHealth intervention.

\section{Study outcomes}

All studies, except Bobrow's study, achieved their primary outcome. Two studies (Piette and Kamal) had short follow-up periods of 6-8 weeks, respectively. Piette's intervention was effective in decreasing blood pressure and Kamal's intervention was effective in increasing adherence to personalised stroke medication. Two studies (Lester and Bobrow) collected follow-up data at 6 months and 12 months. Lester's study showed effectiveness of self-reported HIV adherence at both 6 and 12 months and improved viral load suppression at 12 months. Bobrow's study found a non-significant reduction in systolic blood pressure control compared with usual care at both 6 and 
12 months. There was significant improvement in their secondary outcome of adherence to collecting medication from the clinic.

\section{Variation in outcomes by context-related patient factors}

In Bobrow's and Lester's studies, the interventions were more effective for patients early in their disease trajectory. The interventions are likely to have given the patients opportunity to establish disease management routines. In Piette's study, the intervention was designed to meet the needs of patients with low literacy and the intervention did have greater effect on this subgroup of patients. The intervention is likely to have increased patient knowledge (from a low level) and so increased opportunity for the patient to engage with managing their disease. The intervention is likely to have challenged beliefs about disease consequences and about their capability to manage their disease leading to increased motivation. Bobrow's study found that patients who benefited the most from the mHealth intervention were those with high personal stress caused by multiple psychosocial stressors. The intervention is likely to have provided a structure to their disease management that was lacking in other aspects of their life, increasing their motivation to engage with disease management yet without intruding into other aspects of life. We acknowledge there are certain subgroups that respond particularly well to mHealth interventions (and were under subanalyses within the studies) and where their monitoring improves and why this may be so. Patients with low literacy/high information needs who require knowledge and support (any information is better than nothing) and older patients who have good blood pressure control and/or have been diagnosed within the past 10 years.

\section{DISCUSSION}

All the interventions were effective in terms of improving adherence to monitoring of the long-term condition for which they were designed (HIV, hypertension and stroke management). The interventions in the four studies included different combinations of intervention components but each one included a reminder. We were able to map all intervention components to RFV and/or COM-B mechanisms. Some interventions focused more on the relationship aspect of care provision and others on motivating the patient. Subgroup analysis within three studies suggests that contextual factors moderate the impact of the interventions. In the studies that we reviewed, these factors were patient related: low literacy, high personal stress and time since diagnosis. We found no evidence of moderation by non-patient contextual factors.

Our findings are consistent with those of other international reviews (some within high-income settings). Three systematic reviews found reminders for disease monitoring improve healthcare processes but with education through voice call and text messages, both health outcomes and care processes improve. ${ }^{18} 2223 \mathrm{~A}$ realist review of mobile phone-based health interventions for non-communicable disease management in sub-Saharan Africa ${ }^{48}$ found that patient related contextual factors influenced the impact of interventions. Similarly, text messaging used to improve adherence to antiretroviral therapy had more effect for those with at least primary education. ${ }^{49}$ Taking account of sociocultural factors has been shown to influence scale-up and sustainability of mHealth interventions. ${ }^{50}$ Reviews have found variation in effect depending on how mechanisms were operationalised. For example, weekly reminders worked better than daily reminders, and messages had more impact when they were linked with patienthealth worker interaction. ${ }^{17} 274951-53$ In our review, we were unable to identify variation in impact related to how the mechanisms were operationalised. Our review found no studies where patients received test results or instructions to adjust medication. In high-income countries, interventions have included these aspects of care. ${ }^{182223}$ Most reviews of use of digital communication with patients in sub-Saharan Africa, comment on the problems of implementation of the communication system, particularly technical and maintenance issues. ${ }^{26-28}{ }^{54-58}$ In our included studies, implementation issues had been resolved.

\section{Strengths and limitations}

A realist approach was methodologically appropriate for our research question. However, we were limited by the design and reporting of the included studies. We do not know whether all of the study authors were aware if their intervention designs included domains of RFV and COM-B as mechanisms of change. Among our included studies, there were no interventions of sufficient similarity to allow comparison of outcomes when used in different contexts. Within each study, the authors did not describe variation in the context in which the intervention was delivered. This limits our ability to critically examine the programme theory and could weaken the explanatory power of our conclusions.

The lack of detail in the included study interventions may mean we incorrectly interpreted how and why the intervention worked. Only one of the three studies included a process evaluation. Interviews with the study authors would have clarified their programme theory for their intervention. This would possibly deepen our analysis by accounting for the socioecological dimensions of behaviour rather than being individually focused. The studies had limited follow-up over time, so sustainability of intervention use and sustainability of effect is unclear. The lack of evidence on sustainability is recognised as a serious problem in mHealth pilots in low-resource settings. ${ }^{59}$ The only contextual factors we identified were those measured as patient characteristics and used in subgroup analyses. Also, we may have missed papers for inclusion if we had included non-English papers and extended our search to nursing databases. 


\section{Policy and practice implications and future research}

Our review suggests there is potential for improving the monitoring of chronic disease in LMICs using two-way digital communication, although close connection with local care provision is important. Behavioural theory can guide the design of mHealth interventions aimed at changing health behaviours. ${ }^{60}$ Wong $e t a l^{33}$ states that realist reviews reveal "what policy makers or practitioners might put in place to change the context or provide resources in such a way as to most likely trigger the right mechanism(s) to produce the desired outcome'. It is important to target interventions where impact is most likely, for example, when a patient has more recently been diagnosed. Other contexts which information based mHealth may contribute are when patients have low literacy and low knowledge about their disease. Patients who are under stressful life situations such as poverty, family bereavement or managing multiple longterm conditions may also be amenable and respond well to mHealth support.

Further research is needed on the tailoring of mHealth interventions to the needs and sociocultural context of the patient. For example, the ${ }^{*}$ STAR2D study ${ }^{61}$ underway has found that tailoring dietary advice to patient context is appropriate. ${ }^{46}$

\section{CONCLUSION}

Although chronic disease management is a major burden on healthcare providers in low-resource contexts, there is very little evidence for the use of mHealth for improving chronic disease monitoring in these settings and what exists is mainly on medication adherence. The interventions that have been evaluated mapped to established behaviour theory though this was not always explicit in their description of their mHealth design. There was little evidence of how context moderated the effect of the intervention, except for contextual factors reflected in patient characteristics such as low literacy, under stress or being early in the disease trajectory.

Acknowledgements We thank Samantha Johnson, Medical Academic Librarian, University of Warwick, UK for support with the review's search strategy; Professor Geoff Wong, University of Oxford, UK for his realist knowledge; Professor Donald Cole, University of Toronto, Canada for his advice early in the review and the journal reviewers for their comments.

Contributors JAW led the review, the analysis and drafted the paper with support throughout from FG, who also helped define the scope of the review. $\mathrm{CH}$ assisted with methodological expertise at the start of the review. JG and FXG-0 read various iterations during the review process, giving guidance and advice. KD quality checked the extracted data. All authors provided feedback on the drafts and read and approved the final manuscript.

Funding This review contributed to a PhD thesis called 'The Vutivi study' (http:// webcat.warwick.ac.uk/record=b3045268 S1), fully funded by the UK's Economic and Social Research Council (ESRC ES/J500203/1). GE Healthcare Ltd. contributed funding towards research fieldwork costs for the Vutivi Study and played no role in shaping the research for the PhD.

Competing interests None declared.

Patient consent Not required.

Provenance and peer review Not commissioned; externally peer reviewed.
Data sharing statement All data will be made available on request.

Open Access This is an Open Access article distributed in accordance with the terms of the Creative Commons Attribution (CC BY 4.0) license, which permits others to distribute, remix, adapt and build upon this work, for commercial use, provided the original work is properly cited. See: http://creativecommons.org/ licenses/by/4.0/

(C) Article author(s) (or their employer(s) unless otherwise stated in the text of the article) 2018. All rights reserved. No commercial use is permitted unless otherwise expressly granted.

\section{REFERENCES}

1. Ad-G A, Agyemang C. Chronic Non-communicable Diseases in Low and Middle-income Countries: CABI, 2015.

2. Beran D, Chappuis F, Cattacin S, et al. The need to focus on primary health care for chronic diseases. Lancet Diabetes Endocrinol 2016;4:731-2.

3. United Nations. Sustainable Development Goals: 17 Goals to Transform our World United Nations 2016. http://www.un.org/ sustainabledevelopment/health/ (accessed 10 Jan 2017).

4. Larsen PD, Lubkin IM. Chronic illness: Impact and intervention: Jones \& Bartlett Learning, 2009

5. Schulman-Green D, Jaser S, Martin F, et al. Processes of selfmanagement in chronic illness. J Nurs Scholarsh 2012;44:136-44.

6. Shippee ND, Shah ND, May CR, et al. Cumulative complexity: a functional, patient-centered model of patient complexity can improve research and practice. J Clin Epidemiol 2012;65:1041-51.

7. Kadirvelu A, Sadasivan S, Ng SH, Sh N. Social support in type II diabetes care: a case of too little, too late. Diabetes Metab Syndr Obes 2012:5:407.

8. Glasziou P, Irwig L, Mant D. Monitoring in chronic disease: a rational approach. BMJ 2005;330:644-8.

9. Hamine S, Gerth-Guyette E, Faulx D, et al. Impact of mHealth chronic disease management on treatment adherence and patient outcomes: a systematic review. J Med Internet Res 2015;17:e52.

10. Gaziano TA, Pagidipati N. Scaling up chronic disease prevention interventions in lower- and middle-income countries. Annu Rev Public Health 2013;34:317-35.

11. Mahomed $\mathrm{OH}$, Asmall S. Development and implementation of an integrated chronic disease model in South Africa: lessons in the management of change through improving the quality of clinical practice. Int J Integr Care 2015;15.

12. Stephani V, Opoku D, Quentin W. A systematic review of randomized controlled trials of mHealth interventions against non-communicable diseases in developing countries. BMC Public Health 2016;16:572.

13. Beratarrechea $A$, Moyano D, Irazola $\mathrm{V}$, et al. mHealth interventions to counter noncommunicable diseases in developing countries: still an uncertain promise. Cardiol Clin 2017;35:13-30.

14. Anstey Watkins JOT, Goudge J, Gómez-Olivé FX, et al. Mobile phone use among patients and health workers to enhance primary healthcare: A qualitative study in rural South Africa. Soc Sci Med 2018;198:139-47.

15. Odendaal WA, Goudge J, Griffiths F, et al. Healthcare workers' perceptions and experience on using mHealth technologies to deliver primary healthcare services: qualitative evidence synthesis. Cochrane Database Syst Rev 2015;2010:1-14.

16. Vasudevan L, Zeller K, Labrique A, et al. Mobile health. Digital health: Springer, 2018:15-25.

17. Bärnighausen $\mathrm{T}$, Chaiyachati $\mathrm{K}$, Chimbindi $\mathrm{N}$, et al. Interventions to increase antiretroviral adherence in sub-Saharan Africa: a systematic review of evaluation studies. Lancet Infect Dis 2011;11:942-51.

18. Cole-Lewis H, Kershaw T. Text messaging as a tool for behavior change in disease prevention and management. Epidemiol Rev 2010;32:56-69.

19. Gurol-Urganci I, de Jongh T, Vodopivec-Jamsek V, et al. Mobile phone messaging reminders for attendance at healthcare appointments. Cochrane Database Syst Rev 2013:CD007458.

20. Yasmin F, Banu B, Zakir SM, et al. Positive influence of short message service and voice call interventions on adherence and health outcomes in case of chronic disease care: a systematic review. BMC Med Inform Decis Mak 2016;16:46.

21. Mbuagbaw L, Mursleen S, Lytvyn L, et al. Mobile phone text messaging interventions for HIV and other chronic diseases: an overview of systematic reviews and framework for evidence transfer. BMC Health Serv Res 2015;15:33.

22. Krishna S, Boren SA, Balas EA. Healthcare via cell phones: a systematic review. Telemed J E Health 2009;15:231-40. 
23. Free C, Phillips G, Galli L, et al. The effectiveness of mobile-health technology-based health behaviour change or disease management interventions for health care consumers: a systematic review. PLoS Med 2013;10:e1001362.

24. van Velthoven MH, Brusamento S, Majeed A, et al. Scope and effectiveness of mobile phone messaging for HIV/AIDS care: a systematic review. Psychol Health Med 2013;18:182-202.

25. Fjeldsoe BS, Marshall AL, Miller YD. Behavior change interventions delivered by mobile telephone short-message service. Am J Prev Med 2009;36:165-73.

26. Bloomfield GS, Vedanthan R, Vasudevan L, et al. Mobile health for non-communicable diseases in Sub-Saharan Africa: a systematic review of the literature and strategic framework for research. Global Health 2014;10:49.

27. Catalani $\mathrm{C}$, Philbrick W, Fraser $\mathrm{H}$, et al. mHealth for HIV treatment \& prevention: a systematic review of the literature. Open AIDS $J$ 2013;7:17-41.

28. Chib A, van Velthoven $\mathrm{MH}$, Car J. mHealth adoption in low-resource environments: a review of the use of mobile healthcare in developing countries. J Health Commun 2015;20:4-34.

29. Greenhalgh T, Wong G, Westhorp G, et al. Protocol--realist and meta-narrative evidence synthesis: evolving standards (RAMESES). BMC Med Res Methodol 2011:11:115.

30. Otte-Trojel T, Wong G. Going beyond systematic reviews: realist and meta-narrative reviews. Stud Health Technol Inform 2016;222:275-87.

31. Wong G, Pawson R, Owen L. Policy guidance on threats to legislative interventions in public health: a realist synthesis. BMC Public Health 2011:11:222.

32. Pawson R, Greenhalgh T, Harvey G, et al. Realist review--a new method of systematic review designed for complex policy interventions. J Health Serv Res Policy 2005;10 Suppl 1:21-34.

33. Wong G, Greenhalgh T, Westhorp G, et al. RAMESES publication standards: realist syntheses. J Adv Nurs 2013;69:1005-22.

34. Rycroft-Malone J, McCormack B, Hutchinson AM, et al. Realis synthesis: illustrating the method for implementation research. Implement Sci 2012;7:33.

35. Michie S, Thomas J, Johnston M, et al. The human behaviourchange project: harnessing the power of artificial intelligence and machine learning for evidence synthesis and interpretation. Implement Sci 2017;12:121.

36. Anstey-Watkins. Understanding the potential role for appropriate digital technological solutions in the innovation of health system design, implementation and normalisation in rural South Africa for both patients and health-workers: A critical exploratory analysis: University of Warwick, 2016. PhD Thesis.

37. Lacouture A, Breton E, Guichard A, et al. The concept of mechanism from a realist approach: a scoping review to facilitate its operationalization in public health program evaluation. Implement Sci 2015:10:1:1.

38. Pawson R, Tilley N. Realistic evaluation: Sage, 1997.

39. Dalkin SM, Greenhalgh J, Jones D, et al. What's in a mechanism? Development of a key concept in realist evaluation. Implement Sci 2015;10:49.

40. Michie S, Richardson M, Johnston M, et al. The behavior change technique taxonomy (v1) of 93 hierarchically clustered techniques: building an international consensus for the reporting of behavior change interventions. Ann Behav Med 2013;46:81-95.

41. Vassilev I, Rowsell A, Pope C, et al. Assessing the implementability of telehealth interventions for self-management support: a realist review. Implement Sci 2015;10:59.

42. Kamal AK, Shaikh Q, Pasha O, et al. A randomized controlled behavioral intervention trial to improve medication adherence in adult stroke patients with prescription tailored Short Messaging Service (SMS)-SMS4Stroke study. BMC Neurol 2015;15:212.

43. Piette JD, Datwani H, Gaudioso S, et al. Hypertension management using mobile technology and home blood pressure monitoring: results of a randomized trial in two low/middle-income countries. Telemed J E Health 2012;18:613-20.

44. Lester RT, Ritvo P, Mills EJ, et al. Effects of a mobile phone short message service on antiretroviral treatment adherence in Kenya (WelTel Kenya1): a randomised trial. Lancet 2010;376:1838-45.

45. Bobrow K, Farmer AJ, Springer D, et al. Mobile phone text messages to support treatment adherence in adults with high blood pressure (SMS-Text Adherence Support [StAR]): a single-blind, randomized trial. Circulation 2016;133:CIRCULATIONAHA.115.017530.

46. Bobrow K, Farmer A, Cishe N, et al. Using the medical research council framework for development and evaluation of complex interventions in a low resource setting to develop a theory-based treatment support intervention delivered via SMS text message to improve blood pressure control. BMC Health Serv Res 2018;18:33.

47. Leon N, Surender R, Bobrow K, et al. Improving treatment adherence for blood pressure lowering via mobile phone SMSmessages in South Africa: a qualitative evaluation of the SMS-text Adherence SuppoRt (StAR) trial. BMC Fam Pract 2015;16:80.

48. Opoku D, Stephani V, Quentin W. A realist review of mobile phone-based health interventions for non-communicable disease management in sub-Saharan Africa. BMC Med 2017;15:24

49. Mbuagbaw L, van der Kop ML, Lester RT, et al. Mobile phone text messages for improving adherence to antiretroviral therapy (ART): an individual patient data meta-analysis of randomised trials. BMJ Open 2013;3:003950.

50. Greenhalgh T, Wherton J, Papoutsi C, et al. Beyond adoption: a new framework for theorizing and evaluating nonadoption, abandonment, and challenges to the scale-up, spread, and sustainability of health and care technologies. J Med Internet Res 2017;19:e367.

51. Horvath T, Azman H, Kennedy GE, et al. Mobile phone text messaging for promoting adherence to antiretroviral therapy in patients with HIV infection (Review). Cochrane Database of Systematic Reviews 2012;2012:009756.

52. Pop-Eleches $\mathrm{C}$, Thirumurthy H, Habyarimana JP, et al. Mobile phone technologies improve adherence to antiretroviral treatment in a resource-limited setting: a randomized controlled trial of text message reminders. AIDS 2011;25:825-34.

53. Lall D, Prabhakaran D. Organization of primary health care for diabetes and hypertension in high, low and middle income countries. Expert Rev Cardiovasc Ther 2014;12:987-95

54. Aranda-Jan CB, Mohutsiwa-Dibe N, Loukanova S. Systematic review on what works, what does not work and why of implementation of mobile health (mHealth) projects in Africa. BMC Public Health 2014:14:188.

55. Leon N, Schneider H. MHealth4CBS in South Africa: a review of the role of mobile phone technology for monitoring and evaluation of community based health services: Medical Research Council of South Africa (MRC): Medical Research Council of South Africa (MRC). Health Systems Research Unit, 2012:1-37.

56. Källander K, Tibenderana JK, Akpogheneta OJ, et al. Mobile health (mHealth) approaches and lessons for increased performance and retention of community health workers in low- and middle-income countries: a review. J Med Internet Res 2013;15:e17.

57. Betjeman TJ, Soghoian SE, Foran MP. mHealth in Sub-Saharan Africa. Int J Telemed Appl 2013;6.

58. Gurman TA, Rubin SE, Roess AA. Effectiveness of mHealth behavior change communication interventions in developing countries: a systematic review of the literature. J Health Commun 2012;17 Suppl 1:82-104

59. Sanner TA, Roland LK, Braa K. From pilot to scale: towards an $\mathrm{mHealth}$ typology for low-resource contexts. Health Policy Technol 2012;1:155-64.

60. Michie S, Yardley L, West R, et al. Developing and evaluating digital interventions to promote behavior change in health and health care: recommendations resulting from an international workshop. J Med Internet Res 2017;19:e232.

61. Farmer A. *STAR2D - Text-messaging for type 2 diabetes adherence support in SAA. 2017 https://www.phc.ox.ac.uk/phctrials/trialportfolio/star-d (accessed on 11 Dec 2017).

62. Northouse PG, Northouse LL. Health communication: strategies for health professionals: appleton and Lange, 1992.

63. Martin LR, Haskard-Zolnierek KB, DiMatteo MR. Book and media reviews. JAMA 2011;305:1.

64. Weinstein N. Human motivation and interpersonal relationships: Springer, 2014.

65. Petty R, Cacioppo JT. Communication and persuasion: central and peripheral routes to attitude change: Springer Science \& Business Media, 2012

66. Rosenstock IM. The health belief model and preventive health behavior. Health Educ Monogr 1974;2:354-86.

67. Ajzen I. The theory of planned behavior. Organ Behav Hum Decis Process 1991:50:179-211.

68. Plass JL, Moreno R, Brünken R. Cognitive load theory. Cambridge: Cambridge University Press, 2010.

69. Locke E, Latham G. Goal-setting theory. Buffalo: University of New York, 1994:159.

70. Bem DJ. Self-perception theory. New York: Academic Press Inc, 1973. 